\title{
Algorithm Development for Distributed Memory Multicomputers Using CONLAB
}

\author{
PETER JACOBSON, BO KÅgSTRÖM, AND MIKAEL RÄNNAR \\ Institute of Information Processing. Unitersity of Lmeå. S-901 87 L meã, Sweden
}

\begin{abstract}
CONLAB (CONcurrent LABoratory) is an environment for developing algorithms for parallel computer architectures and for simulating different parallel architectures. A user can experimentally verify and obtain a picture of the real performance of a parallel algorithm executing on a simulated target architecture. CONLAB gives a high-level support for expressing computations and communications in a distributed memory multicomputer (DMM) environment. A development methodology for DMM algorithms that is based on different levels of abstraction of the problem, the target architecture, and the CONLAB language itself is presented and illustrated with two examples. Simulation results for and real experiments on the Intel iPSC/2 hypercube are presented. Because CONLAB is developed to run on uniprocessor UNIX workstations, it is an educational tool that offers interactive (simulated) parallel computing to a wide audience. (c) 1993 by John Wiley \& Sons, Inc.
\end{abstract}

\section{INTRODUCTION}

Todar. much algorithm design for parallel computer architectures and most implementations are done in conventional programming languages like Fortran and (:. Nomally. this is a very timeconsuming process. especially in an innovative phase where different ideas and prototype implementations are examined. It would be desirable to be able to express the computations in as high level of abstraction as possible and to focus on the parallelization issues and problems for different architectures. The application area we have in mind is marix computations that are basic in most scientific. economic. and engrineering appli-

Rocenived Mar 1902

Acrepted dovember 1902

(c) 1993 by John Wiley d Sums. Ine.

Seintidic Programming. Vol. 1. pp. 185-2(1)3 19(1)-2

(:c:(: $1058-92+t / 9.3 / 0201855-19$ cations. It is well known that block algorithms are amenable for many parallel architectures [1-3]. Good interactive development environments for sequential computations exist. notably VATLAB MATrix LABoratory [t]. MATLAB has simple operators and built-in functions for matrix addition and multiplications. matrix factorizations. etc. This paper presents the CONLAB environment with focus on algorithm development for distributed memory multicomputers (DIII).

CONLAB (CONcurrent LABoratory) is an enviromment for developing algorithms for parallel computer architectures and for simulating different parallel architectures. This means that the user can experimentally verify and obtain a good picture of the real performance of a parallel algorithm executing on a simulated target architecture. The aim with CONLAB is at leas twolold. namely, to provide an environment for developing and testing parallel algorithms. and an educational tool for introducing parallel computing in both teaching and research. Because CONLAB is 
developed to run on uniprocessor L NIX worksations it offers interactive simulated parallel computing to a wide audience. At present. CONLAB is mainly focused on algorithm design for. and simulation of. DMII architectures with message passing communication.

CONLAB gives a high-level support for expressing computations and communications in a DMM environment. Section 2 gives an introduction to the CONLAB langruage. the simulation of parallel execution in CONL.AB. and different tools related to parallel algorithm desirn and simulation of DWMI architectures. Section 3 introduces a development methodology for DMM algorithms that is based on different levels of abstraction of the problem. the target architecture and the CONLAB language itself. In section 4 . the development methodology using CONLAB is illustrated with two examples. Simulation results for and real $\mathrm{x}-$ periments on the Intel iPSC/2 hypercube are presented in section 5 . Finally, in section 6 some conclusions are given and the future development of CONLAB is discussed.

\section{THE CONLAB ENVIRONMENT}

CONLAB has inherited many characteristics from MATLAB [t] It is an interactive environment for scientific and engineering computations that also offers parallelism. The language used for programming in CONLAB is similar to the VATLAB language (in reality a subset because all facilities of MATLAB are not implemented and is extended with constructs used for expressing parallelism, synchronization, and communication. In the discussions and descriptions that follon we assume that the reader has some familiarity with. and experience in, using VATLAB $t$ or a MATLAB-like emironment. As in MATLAB the only data structure are matrices (scalars are $1 \times 1$. vectors are $1 \times n$ [row vectors] or $m \times 1$ [column vectors]. and matrices. e.g., $m \times n$ ). The colon notation is used to specify columns. rows. or submatrices of a matrix $A$. For example. $A(: i)$ denotes the $i$ : th column of $A$ and $A(i: j, k: l)$ denotes the entries of $A$ in rons $i$ through $j$ and columns $k$ through $l$. We will frequently refer to and use concepts like predefined and user-defined functions. different matrix operations, etc.

Because CONLAB is an interactive environment it frees the user from issues like compilation, linking, etc. The user can define a function in- teratively and call it immediately. In the same way a process bee section 2.2 can be defined. assigned to a set of processors. and simulated parallel execution can be started insantaneously,

In the following we give an introduction to the parallel concepts of the CONLAB languare. the simulation of parallel execurion in CONLAB. ant different toolis related to parallel algorithm design and simulation of DMII architectures. lllustrations of their use are shown in two examples of section 4 . Other reports provide more information about CONLAB [5-?].

\subsection{Where Does CONLAB Run?}

CONLAB is mainly intended to run on uniprocessor C XIX work-tations. bu it could profitably be ported to muliprocesior workstations or even supercomputers to achieve better porformance of big simulations. The computational part of CONLAB is based on the high-performance linear alectra package LAPACK $[3]$, which is portable to many of today adranced computer architectures. In fact by running CONLAB on a very powerful machine it is possible to simulate the actual computations faster than running the same application on the DMII target machine (at least for the first and second generations D.MII architectures.

\subsection{Extensions for Parallelism}

Parallelism is expressed with a new control structure. the process. A process is very similar to a function. in that it is a named collection of statements that can be initialized with a set of arguments. A process can be asigned to one or more virtual processors. and parallel execuiron of these processes can be simulated by time sharing on a LNIX workstation. Arguments can be passed to the process when it is assigned and they are typically used for different initializations.

Communication via message passing is achieved with send and receive primitives. A message can be sent to one or more processes and a trpe is specified for the message. Reception of messages can be done by specifying the sender and/or message type. Communication can be either sunchronous or asynchronous. When sinchronous communication is used the sender wats until the message is completely received by the receiver. whereas for asynchronous communication the sender continues execurion immediately after submitting the message. 


\subsection{Simulation of Parallel Execution}

The simulation in CONLAB is hased on a stack mathine. The CONLAB statements are compiled to a prendo code that is exeruted by a virtual sack machine. The values on the sack are matrires. which are the only data type in CONL.AB. and the mathine instructions perform matrix geerations on the stack elements.

The processes share the CPL of the simulator and exerute a series of wate machine instructions perating on their priate high-level arak.

To control the simulated parallel execution of the procesnes all procesises arte provided with a time value, which describes her lar in exerduion a

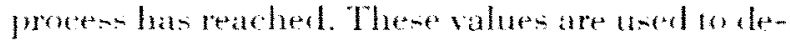
termine the scheduling of the processes as weth as in contol the snchronization of the prowestes. For each stark machine instruction a procesen execultes. it time value is increased according 10 a time model. dercribed in section 2.t.

Paraltel execution of processes is ardheved by letting the processes execute in a time shared manner. Duringr one lime slice a process is allowed 10 execute a few stark machine instructions. operating on its own private stack. at long as it does not execute instructions with side effects e.g. communications. Because a side effect is dependent on and changes the qublal state of the syotem of procenses. a process that executes an insmuetion with side effects must be the process with the loxent virtual simulated time value. Ohemise it has to wait for the other processes " ("atching up," in virual time $[?$. Alter one time flice another process is selected for execotion. and the new aretive process operates on its private stack. and oo ain.

Alter each instruction a process executes. at new rime value is computed for the process. This time value is checked against the time tice and the process is allowed to montinue execution as long as the times value does not exceed the time sice.

At each step the procesis with the smallest time value is selected for execution. This ensures that all processes will eventually bo allowed to execute. provided that they perfom floating point operations and/or communication.

\subsection{The Time Model for Simulation of DMM Architectures}

The execution of the procesies on the virual processors is controlled by a time model that com- putes the elaped time on earh virual processor. The model is divided into t wo parts. compunations and conmunications. Differten DNII architerures an be simulated by changing his time menlel.

The tine model for eomputations is hased on the number of llowing peint operations thops: a procese performs. and the cost for one flop. In this comext multiplications as well as additions. subtractons. and divinions are considered at floating print operations with the rame cost. The daes specities with a call to the finction floptime. the cost for one floating poine operation. Typically. this value represents an aterage of the practicat performance of the note prosenort. Fach time a computation is performed by a process the new time value. taral. of that proceson is computed as

$$
t w a l=t a d+\# f l o p s \cdot t_{\text {mop }} .
$$

where $t_{\text {hon, }}$ in the time cost associded with a floating point operation.

The time model for communications on DMII architectures is based on the faret that on a real DIMI. communication ean take different seenarios depending on the relative order in which the sending and receiving nodes issue the send and receive calls, respectively.

1. Linbulfered communication is the result when the receiving node has isued a receive call before the message has arrived at the nodt. When this happens the communication syom knows where wo put the message and no communication buffers have to be uned.

2. Buffered communication oceurs when the receiver has not performed the receive call when the message arrives. Becanse the communication ststem cannot know where in the user data area the message is to be placed in is forced to use intemal buffers.

The communication time model has the following components:

1. Send time is the time it takes for the sender of the message to contact the local conmunication system. The send time includes copving the message from the local data area to the communication system buffers. The send time is divided into bulfered send time and unbulfered send time to disin- 


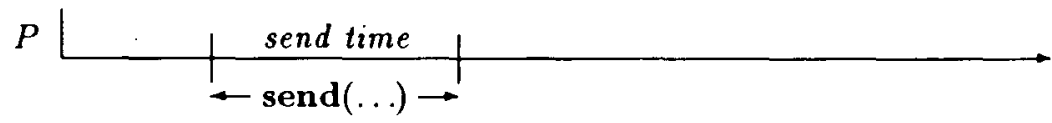

time

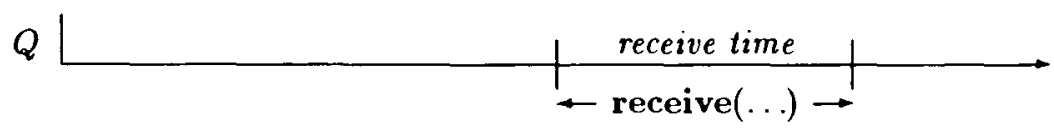

FIGURE 1 Communication time components for buffered communication.

guish between the two types of communication.

2. Receive time is the time it takes for the receiver to deal with a message that has arrived at the node. Note that receive tine only concerns buffered communication.

3. Delay time is the time it takes for the message to reach the target node after the sender has initiated the send operation.

4. Total communication time. When unbulfered communication is used the receiver is blocked on receive when the message arrives. The total communication time for unbuffered communication is the time it takes for the message to be fully transmitted to the receiver from the point where the sender issued the send rall.

The following small program illustrates the communication time components.

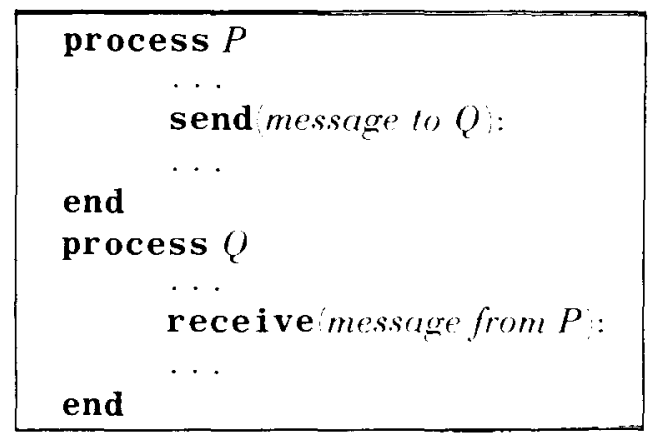

The process $P$ sends a message to the process $Q$. using the send statement. Process $Q$ receives the message from the process $P$. with the receive call.

If $Q$ initiates the receive after $P$ has made the call to send then buffered communication is used. Figure 1 shows the relationships between the time components when buffered communication is achieved in the above program.

If the receive call is executed before the call to send then unbuffered communication is used. which is depicted in Figure 2. In Figure 2 the delay time decides when the receiver changes state from idle. waiting for a message to active in communication. During simulation the delay time is used to determine if the message has reached the receiving node by the time the receive call is done. This information is used to decide if buffered or unbulfered communication is to be accomplished.

The communication time components are presented to the CONLAB simulator by specifying a function expressed in CONLAB notation. This function is implicitly called every time a communication is initiated by a send call and it is responsible for computing the tine components described above. The function takes the menoage size and type and the sender and receiver procesises as arguments and delivers the send. receive. delat. and tolal eommunication times as results. The time components are then used to calculate new time
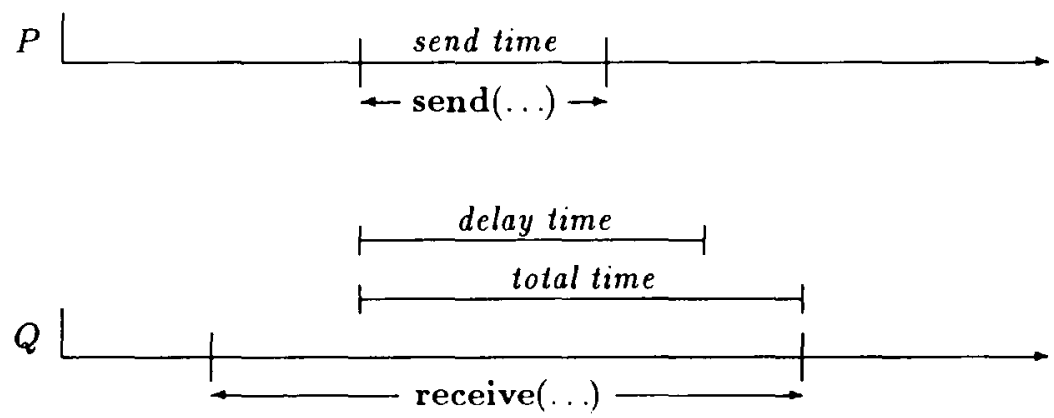

time

FIGURE 2 Communication time components for unbuffered communication. 


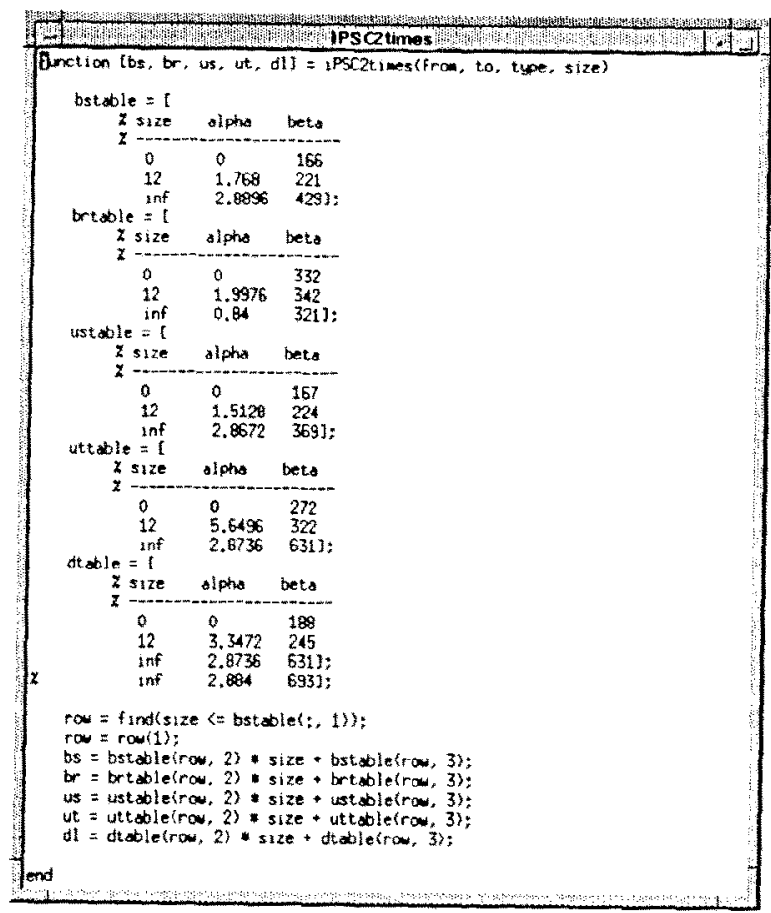

FIGLRE 3 CONL AB function for computing rommunication time components for the ipsc/2 byese'nile.

values for the proceses involved in the eommuncation.

Figure 3 shows an example of a $60 \times L+B$ function that computes the communication time components of the lnel ilsc:/2 hypercule. The tine components for bulfered communication bs, br and unbuffered communication us, ut, dl atre approximated by the unaal linear model

$$
l_{1 \cdots+1 ! \cdots+\cdots) t}=\alpha \cdot y+\beta
$$

where $\beta$ and $\alpha$ demore the sant-up cos and the per-umit cosi lor transferring a mesiage of wae $/ 1$ donble words 8 bytes. Mepectively. Detailed communiration henchmarks resuling in this time model for communication is described in $[9$.

By changing the value of floptime and/or the tables of the communiation time function. the uner can. for example examine different computation-to-communication ratios of a DMII medel. Differen D.MII ardhtectures can be simulated by nriting new functions for computing communication time components. The time moled for compuations does non incorporate the simulation of memory hie rarchies. It would then be necessars of extend floptime to a function that approximates different components as in the communication time model (e.g. cache effects, pipe-lining).

\subsection{Performance Measuring}

The functions timer, arithtime. and commtime are used to calculate the timing characteristics of a CONLAB program. The timer function returns the current time value of a process it is initialized to zero when the process sturts and it can be used to measure the elapsed execution time of a process. similarly. the functions arithtime and commlime return the current times for arithmetic computations and communications. respectively boh initialized to zero when the process starts.

The arithmetic lime can also be computed as floptime flops. where the function flops returns the number of floating point operations performed by the process.

\subsection{Monitoring Process Status During Simulation}

During simulation the user can oltain inlomation about the status of all procestes. Process uilization the number of husy processes and process satus busy or waingl can he plotted as a function of time se Figr. $t$. Processes in busy state are marked in black green on a color screen: while processes in waiting or idle states are marked in white red.

\subsection{Animated Replay of Messages}

As an option CONLAB will generate wave hles of all communications and can therefore offer the user a way of replaving the simulation in terms of communieation by message pasting. This replay shons sending and receiving processes. respectively. for earh mesiage that was tranferred. It also thow the messiage type and size and the rimes the message was sent and received. The contents of the message can also be printed out. In this way the mesiage replay is a tool for debuerfoing the communication of a listributed algorithm. Before the replay starts. the user has the option wo sort the messages by send time or receive time. The mesage replay window is shown in Firure 5. This nindow dump shows that note 11 is receiving a mesiage of type 2 and length 250 bres: from node 8. 


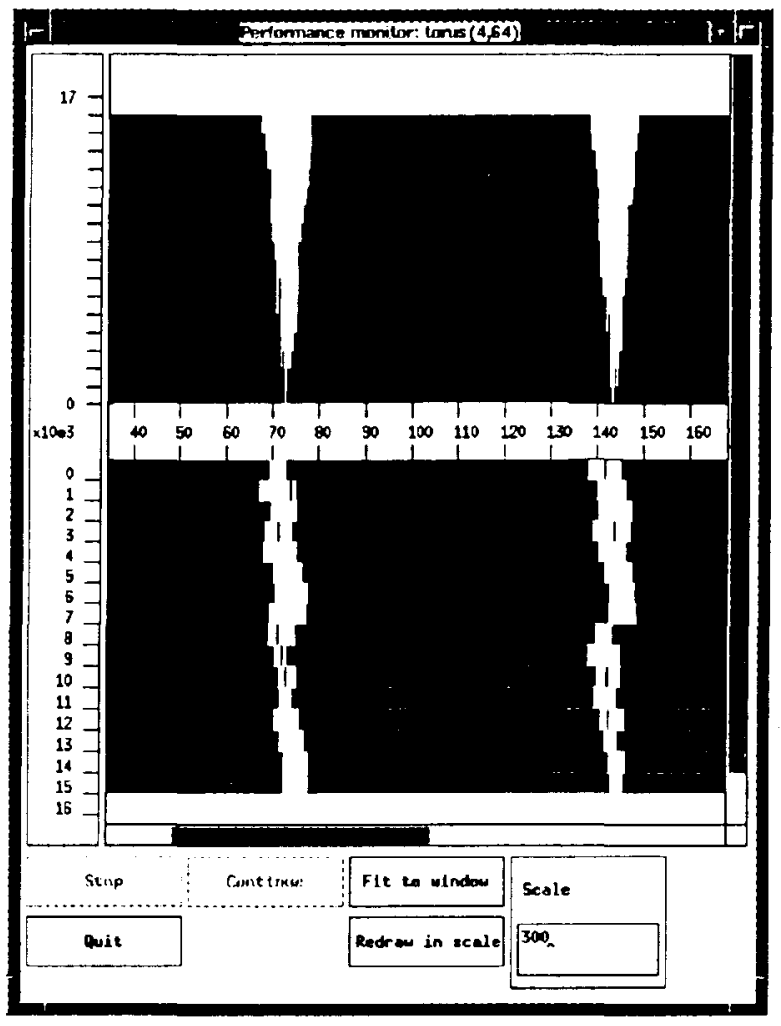

FIGURE 4 The performance utilization (perf) window.

\subsection{ParaGraph}

There is a possibility to use trace files generated by CONLAB in the visualization tool ParaGraph [9]. ParaGraph, which primarily is intended as a postprocessor to the instrumentation package PICL $[10]$, includes several graphs describing algorithm performance when used with CONLAB

\section{ALGORITHM DEVELOPMENT METHODOLOGY}

The methodology for developing parallel algorithms in CONLAB is based on different levels of abstraction of the problem. the target parallel architecture, and of the CONLAB language itself. $\mathrm{By}$ following the methodology the user will implement, at as high abstraction level as possible, functions and processes in CONLAB that define the architecture topology. communication on the topology, node and host algorithms, and a user interface. Below, we describe the different levels of abstraction.

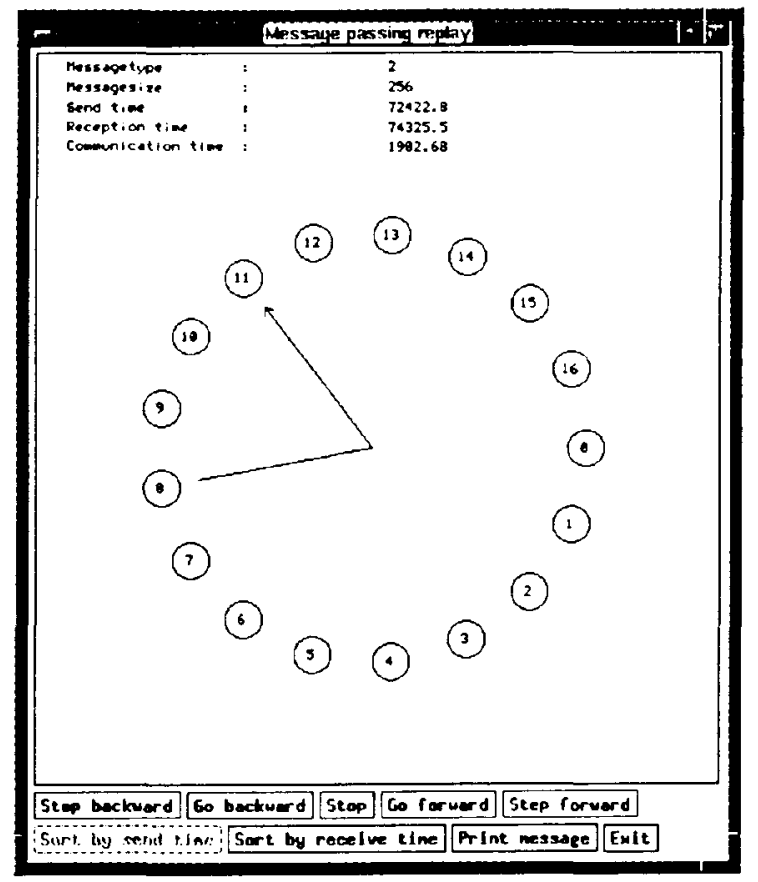

FIGURE 5 The messagr replay replay, window.

1. The first level of abstraction concerns process topology functions. These functions describe the topology of the targer architecture (real or virtual, and are used to navigate in the topology. For example, process topology functions in a two-dimensional mesh translate a two-dimensional processor index into a single process number and vice versa. Typically. a binary reflected Gray code numbering of the procesiors in a topology e.g. ring. mesh. or hypercube, is used to assure that logical adjacent processors are also physical neighbors. Other topology functions may deliver the physical neighbors in the four directions of a twodimensional mesh (north. south. east. and west).

2. The second level concerns communication functions. These functions are problem dependent but can of course be general. Typically, they perform some kind of compound communication on the target architecture. for example, rolling messages row-wise in a two-dimensional mesh or broadcasting a message to a set of nodes. The communication functions use the process topology functions to direct the messages and the send and receive primitives in the CON- 
LAB language to execule the intended communication by message pasing.

3. The third level delines the node proces- dhat implements the distributed algorithm. Typically. a node program sarts by receiving problem data from the host see the next level before entering a loop comprising compuations and internode communications. Finalls, the penduls are delivered to the hom process. Computations are expressed in the high level constructs and operations aflered by the CONLAB language. Communications atre expresined by using the communication functions.

t. The fourdh level defines the hat process that starts the node processes ly an-igning them to virtual procesenors. Typically. the host process distribues problem data to the node proceseses and collects results at the end of the execution. Also the host-1o-node communication and vice versa) is expressed in terms of the communicution functions.

5. The fifth and topmost level is the simulation function that defines the interface to the CONLAB user. annigns the hont process to a virtual processor. and starts the simulation. Typically. the simulation function communicates problem and architecture parameters e.g.. problem size. input data. and the number of processors!

Concepually. the described development methodolog is well known and hopefully used by most algorithm designers for D.M arehitectures. The novelty is that CONLAB gives a high-level suppon for expressing the computations and defining topology and communication functions. CONLAB is extensible in the sense that functions and processes once defined will afterwards exist within the emvironment and can be used similarly to predefined functions.

The host and node processes define the distril)uted algorithm. which is a composition of communicating processes. The concept of a host process and node processes is motivated by the commercially available DMM architectures. and in CONLAB, they are considered equal. Within each process all computations are sequential and performed within a single address space (the local memory of the node).

The simulation function can be seen as the problem (or application) level of the abstraction. By changing problem sizes and/or the number of procesonors the user can eraluate the parallel performance of the distributed algorithm for the -imulated Gralable D.III architecture.

See aloo the $X Y Z$ ahstraction levels defined by snyder [11.12 where the X-ferel is formed by the sequential operations performed within a process. Phases, the parallel omposition of processes. define the $\mathrm{Y}$-level. Finally. problems. the componition of phases. define the Z-level

\section{TWO EXAMPLES OF ALGORITHM DEVELOPMENT FOR DMM ARCHITECTURES}

In thim -ection ne illustrate the algorithm development methodology for D.MII architectures deseribed in the previous section. with wo examples. The lims example shows bow a distributed algorithm for block matrix muliplication on a torus connected awo-dimensional mesh of processors is developed and implemented in CONLAB. The second example shows how an already existing serial algorithm for a block QR factorization. expresied as a MATLAB function. * can be parallelized ino a ring-oriented distributed block algorithm in CONLAB.

\subsection{Block Torus Matrix Multiplication}

Given $n \times n$ matrices $A$. $B$ we will compute $C=A$. $B$ on a txo-dinensional mesh of $p$ processors $(p=$ $s \cdot s$ with torus connectivity. To simplify the notation we assume that $n=n b \cdot s$. that is. $A$. $B$ and $C$ have $s^{2}$ blocks denoted $A_{i j} . B_{i j}$ (and $C_{i j}$. respectively, of size $n b \times n b$. Otheraise. the last block column and block row of the matrices will have rectangular block:.

The serial ijk-algorithm for block matrix multiplication block inner-product looks as follows.

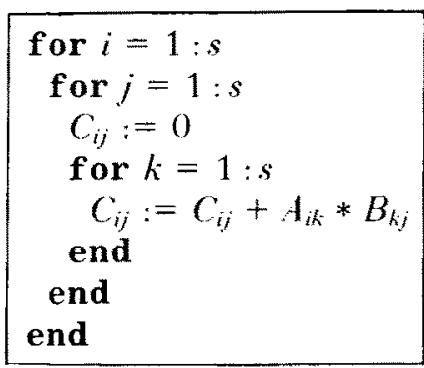

In the distributed algorithm the processors are organized in a two-dimensional mesh where com-

* MATI AB and CONLAB functions have the same syntax so the sample function could also have been developed in CONLAB 
munication takes place between nearest neighbors as well as around the edgre in both directions. In order to compute $C_{i j}$ at node $(i, j)$ we need access to block row $i$ of $A$ and block column $j$ of $B$.

Initially, the matrices $A$ and $B$ are distributed block-wise among the nodes in the mesh so that node $(i, j)$ has the blocks $A_{i k}$ and $B_{k j}$. where $k=$ $(i+j-2) \bmod s+1$. This means that the matrix $A$ is skewed row-wise and $B$ is skered columnwise. This distribution scheme allows node $(i . j)$ to compute $C_{i j}$ with only nearest-neighbor communication in the two-dimensional mesh. The main steps of the algorithm [13] for node (i. $j$ ) look as follows.

$$
\begin{aligned}
& C_{i j}:=0 \\
& \text { for } t=1: s \\
& \text { Receive } A_{i k} \text { and } B_{k j} \text {, where } k=(i+j+t-3) \text { mod } s+1 \\
& C_{i j}:=C_{i j}+A_{i k} * B_{k j} \\
& \text { Send } A_{i k} \text { to nearest neighbor to the west } \\
& \text { Send } B_{k j} \text { to nearest neighbor to the north } \\
& \text { end }
\end{aligned}
$$

Notice, the first time node $(i, j)$ receives an $A$ block and a $B$-block they are delivered by the host. but the following blocks originate from the nodes to the east and south. respectively.

It would also be possible to let node $(i, j)$ initially hold blocks $A_{i j}$ and $B_{i j}$. However. this would impose some initial redistribution of the block: into the skewed pattern described above. In the node algorithm above, we assume that the host process will effect this skened distribution at once.

Figures 6 through 10 describe the structure of the complete CONLAB program. The topologry functions (see Firg. 6) are coordenode. norde2 coord which convert coordinates in the two-dimensional mesh into a node number obtained by the predefined CONLAB function getpid and vice versa. and north and west which deliver the node number of the neighbors to the north and west. respectively. They use the functions gray. and gine, which retum the gray and inerat gra! codes. respectively. The communication function is roll inep Fig. 6). which sendo a mesiagre in a specified direction of the two-dimensional mesh and receives a new mensage from the opposite direction. In Figures? and 8 the CONLAB program for the node process and an accompanying header file torus.h are displaved. Figure 9 shows the CONLAB program for the host process. Besides the header file, the host process also includes the file block. $h$. which conains the maros tor the antomatic matrix blocking facility $[6 \%$ Votice the distribution of $A, B$ to the nodes and the reception of $C$ could also have been defined as communieation functions. Finally, the simulation function with the parameters $A . B$ and the size of the twodimensional mesh is displayed in Figure 10.

\subsection{Ring-Oriented Block QR Factorization}

In this example we will sep how an existing YATLAB program can be paralle lized and implemented in CONLAB. We comsider a block aligorithm to perform a $Q R$ factorization of an $m \times n$ matrix A. that is

$$
A=() \cdot R
$$

where the $m \times m$ matrix $Q$ is orthogemal and the $m \times n$ matrix $R$ is upper triangular trapezidal. The $Q R$ fatorization can be computes in several ways. for example. be utilizing hock Householder transformations [ 14 . A block Householder transformation $Q_{i}$ is a product of $n b=$ the block size Householder matries. Heare ne only consider one block algorithm that is bated on the 11 I reprementation of block Householder transformations $Q_{i}=$ $\left.I+I I^{T}\right)[1+$. The main steps of the algorithm are summarized below:

$Q=I$. the identity matrix of order $m$

$n b l:=n / n b$, number of column blocks awh nb columns: in $t$

for $i=1: n b l$

Find $I I$ and $Y$ such that $\left.Q_{i}=I+I I\right\}^{T}$ zeroes block colum

$i$ of $A$ below the diagonal (columns $[i-1 ;-n b+1: i \cdot n b !$.

Apply $Q_{i}$ to the remaining colum blocks of $A$

$Q:=Q \cdot Q_{i}$

end 


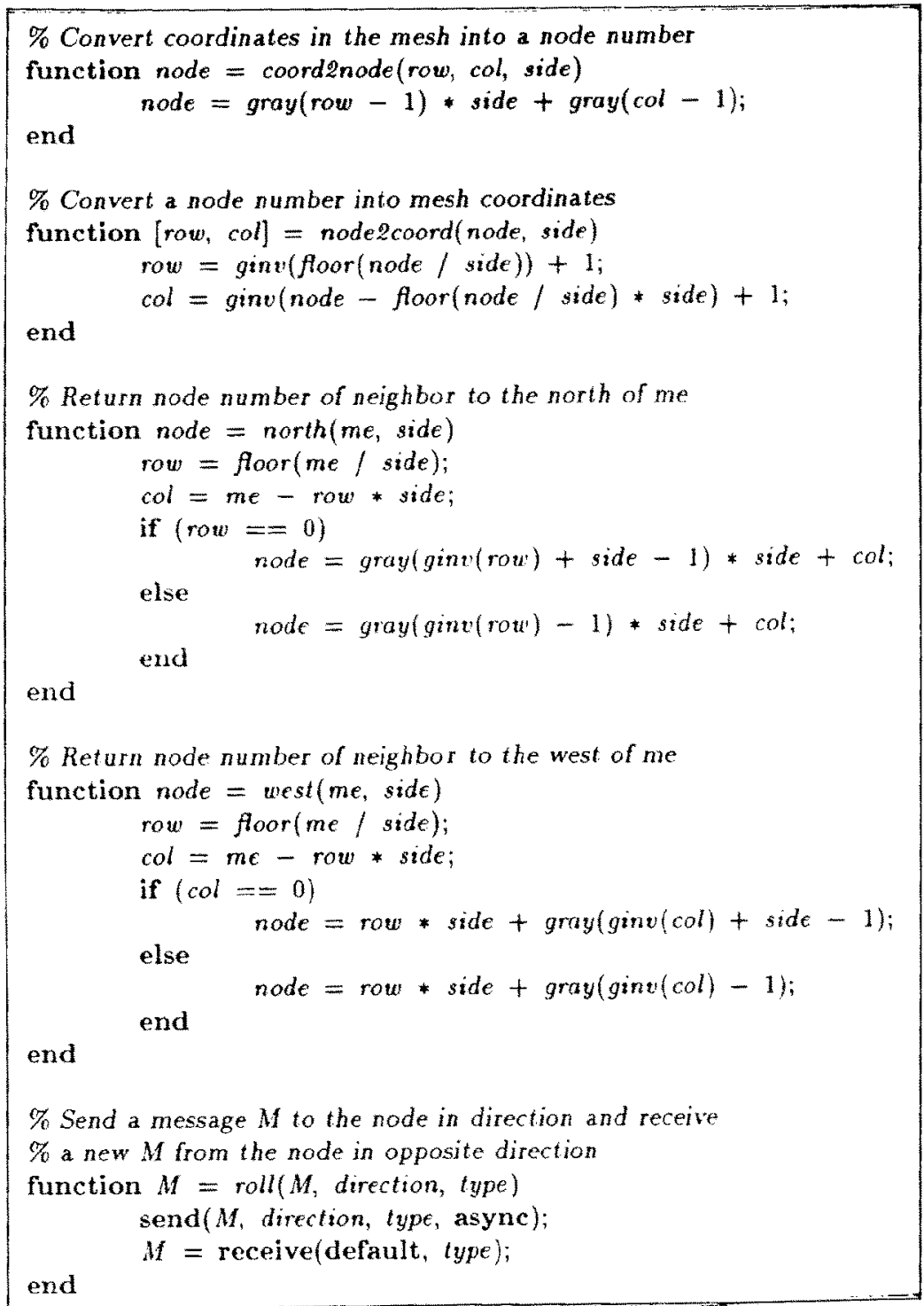

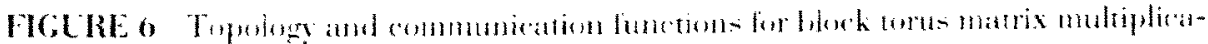
tiin.

The YATLAB function grt for this algorichm is shown in Figure 11.

It is eaty to see that the applifation of $Q_{1}$, any

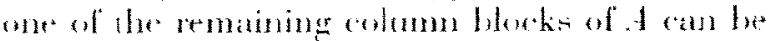
done imlepententy and the data llow an the blenck

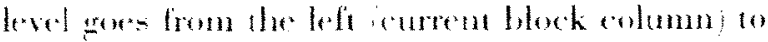
the right remaining hock rolumms in a linear list. If we partition data no that each node in the list

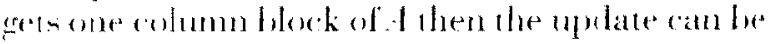
dome in parallel. that is. earh nerde upelattes its own column bleck of 1 with (). Our lirs attempt to a distributed algorithm apese as follows: note 1

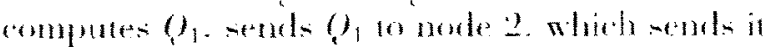
to moke 3 . and no on. Vorke 2 through p then up- date their column blocks independenty and in parallel. Next the procedure is repeated but we start winh node 2 instead of note 1 . and so on until all nodes have compuned their pant of $Q$ atmel $R$. The olvious drawhack with his algorithm is that after the first um note 1 is idle and so an. The remedy in to let each note have more than one columm block and nrap-map them so that node i hold column blocks $i . i+p . i+2 p$ and $i$ or on where $p$ is the number of procesons This gives us a ring topologe with a much beter lowal balaneing of the computational work. Now, the main steps of the node algorithm looks as follows. 


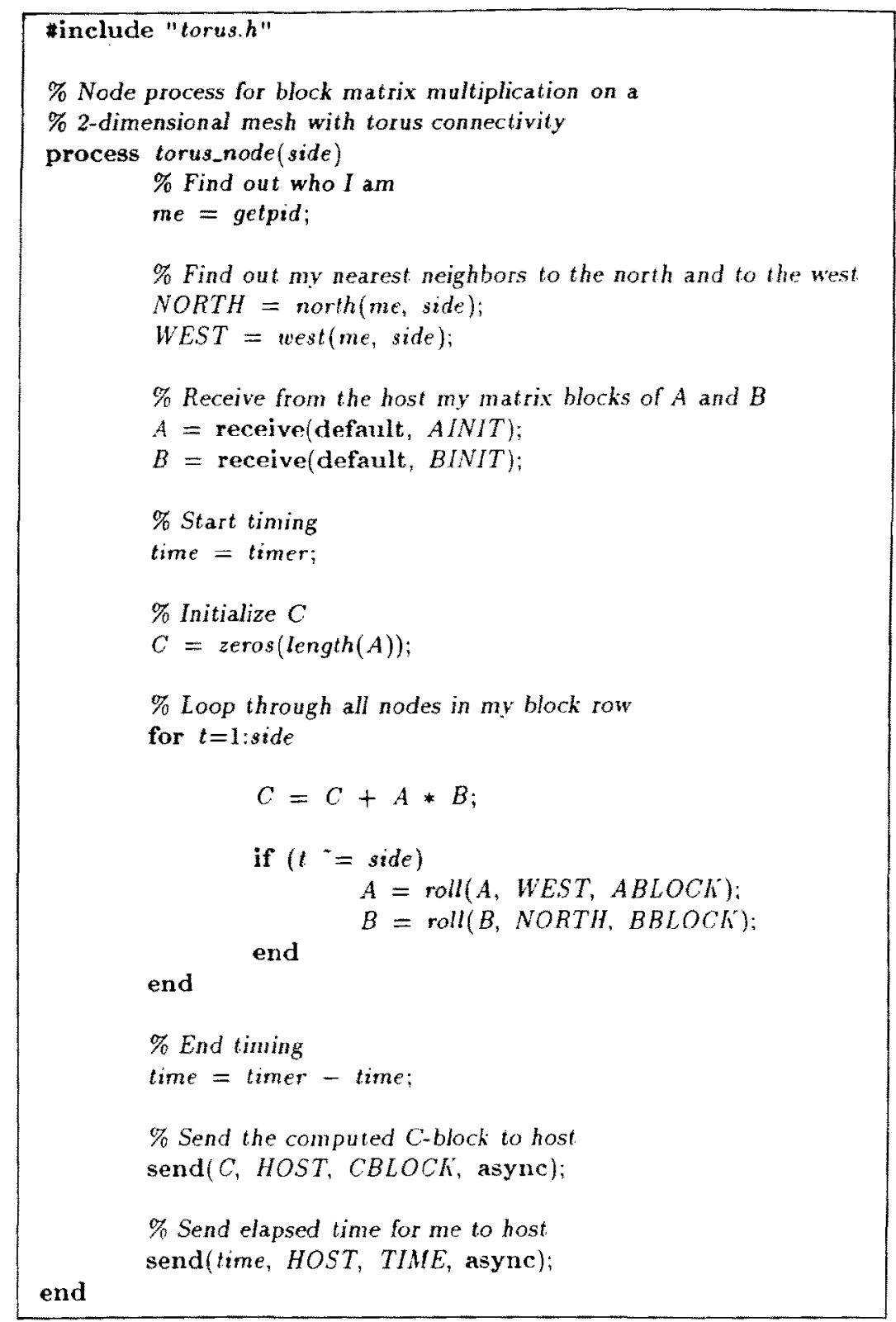

FIGLRE 7 Vode program for block torus matrix multiplicatim.

$l b l=0$. counter for the number of blocks my node holds

nexttogenerate $=1$. keeps track of which process renerates the next transformation for $i=1: n b l$

if I am nexttogenerate

1. $|b l=| b l+1$

2. Compute $I / Y$ factors for my colunn block $l b l$

3. Send $I$ and $Y$ to my right neighbor

else

4. Receive $I V$ factors from my left neighbor and send it further end

5. Update my remaining column blocks of $A$ with the new $U$ and $Y$ end

6. Lpdate my row blocks of $Q$ with the new $I$ and $r$ 


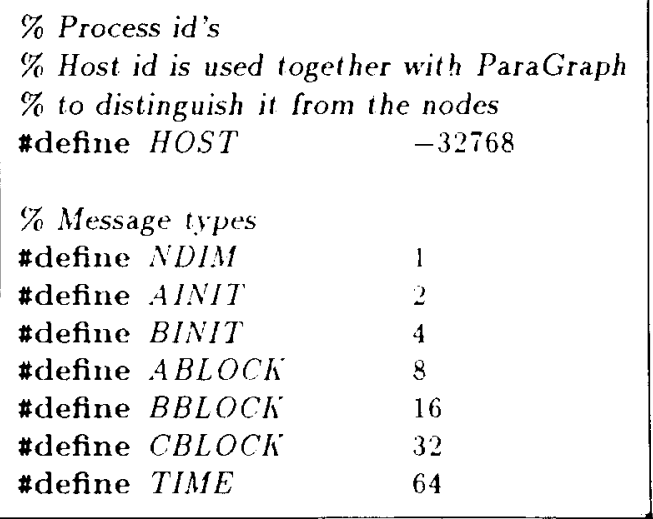

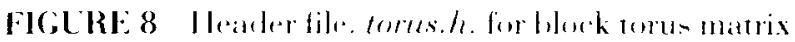
muttiplieations.

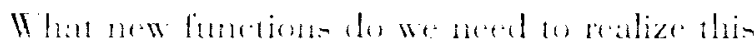
alererithm? In -tepe 2 we nexed a lunction that computes $/ /$ and $/$. hut this lumetion aldeady exist in the serial algorithm hesheg for Householder block getenterator

In - tepe 3 and + we need to communicate nith the left and right neighlors in the ring. (one simple wall of doing this is to number the nodes from 0 on $p-1$ and then the lefi and right neighteor have the node numbers me -1 and me +1 . respece tively. The topoloce functions left and right are shown in Figure 12. Communication functions (listh and rect for di-nibuting and receiving hlork nrap-mapped data are shown in Figure 1:3. For example. the function recb receives column or row block- of a matrix distributed with column cor row block nap-mapping and packs them into a lencal array.

sleps 5 and 0 are basically the same an in the serial algorithm. Tow. we only apply the II facewor- wo the blocks of t and () that each node holds. The CONLAB program for the node procest comprising tono parts and aceompanying header file are shown in Figures 1+-10. By filerek nrapmapping $Q$ row-wise the update of $Q$ is similar to the serial algorithm are Figs. 11 and 15). For receiving block wrap-mapped data from the hose ne use the communication function rect see Figr. 13). The host program is straightforward: it assigns the node program to the different nodes. starts the simulation. distributes $t$ and (). and tinally. receives the results from the nodes. The distribution of $t$ and $Q$ is effected by using the communication function distb see Fig. 13. Figure 1? shows the CONLAB code for the hos process. Finally. the simulation function qrb_sim with parameters 4 . the block size of $t$ and the size of the ring is displayed in Figure 18.

\section{SIMULATION RESULTS FOR AND REAL EXPERIMENTS ON THE INTEL IPSC/2}

The two algorithms presented in section thave also been implemented in (: block torus matrix multiplication and Fortran ring-oriented bleck QR lactorization and lested on our Intel iPst:/2 hypercube. The machine has of salar s. nodes. rach equipped with a $16.11 \mathrm{~Hz}$ Intel 800380 processor with + Jlhye memory and a Weitek 116 ? SX with a theoretical peat performance just under 0.6 Vllope in double precision real ariblumetic. The iPsi: 2 communication module use- a di-

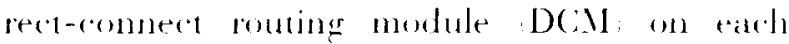
node. with a bandwidth of 2.8 . Yluges/ here in each direction. Detailed communication benchmarks and molels defining the communication time funtion in (CO)LAB finthe iPSC: 2 are presented Iy Jacoboon $[8$ see Fig. :3.

Table 1 show the real and simulated results for the block torum matrix muluplication. Tathe 2 shows similar results for the ring-oriented block ()R factorization. In Talses 1 and 2 we use the following notation: $n=m$, the matrix size. $p$ the number of procenorors. $T_{p}$, the parallet time in seces for p procensors. St, the parallel opeerlup computed as $T_{p} / T_{1} . E_{p}$, the parallel efficiency computed as $S_{\mu} / p$.

The results for the ring-oriented block ( $/ R$ facwrization agree almos completely. whereas the results for the torus matrix multiplication do not. The main reason is the super-linear speedup of whe block worus algorithm on the iPsi/2. which is hard to understand completely the node processors are utilized more efficiently for smaller matricest. CONLAB underestimates the execution time on one procesons. resulting in too persimistic speedup lactors for 4.16 . and of procesons. However. the resulis from CONL AB correspond to what is expected in theory: for fixed number of processors the parallel efficiency increases as a function of the problem size but keeps below 1.0 . The time model makes it impossible wobtain super-linear speedup in CONLAB.

Figures 19 and 20 show window dumps from ParaGraph displaying characteristics of the executions of the ring-oriented block $Q R$ algorithm on the iPSC:/2 and in CONLAB. The Spacetime diagrams show the communication paths. where a line between two nodes indicates communication. a horizontal line indicates computation. and the absence of a horizontal line means that the processor is idle. Notice that the implementations are not identical. On the iPSC/2 the ring is ordered with Gray code numbering. whereas the ring in the 


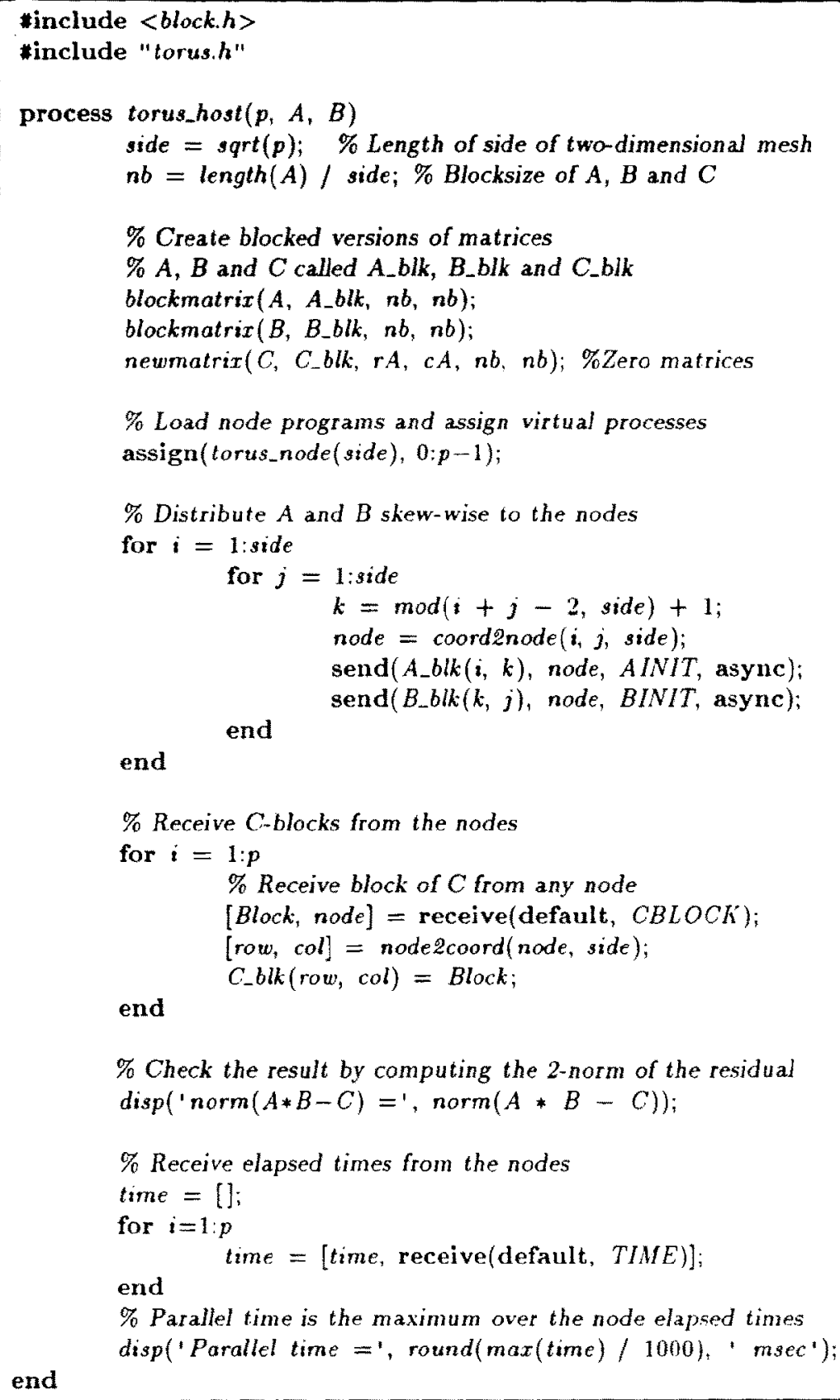

\% Check the result by computing the 2-norm of the residual $\operatorname{disp}(' \operatorname{norm}(A * B-C)=$ ', $\operatorname{norm}(A * B-C))$;

\% Receive elapsed times from the nodes time $=[]$

for $i=1: p$ time $=[$ time, receive $($ default,$T I M E)] ;$

end

\% Parallel time is the maximum over the node elapsed times disp('Parallel time $=$ ', round $(\max ($ time $) / 1000)$. 'msec');

end

FIGLRE 9 Host program for block torus matrix multiplication.

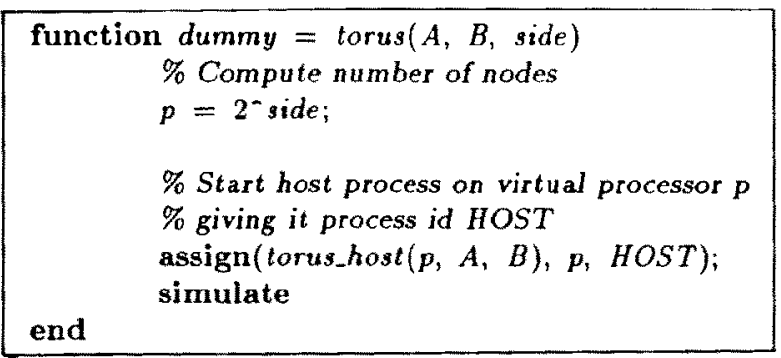

FIGURE 10 Simulation function for block torus matrix multiplication. 


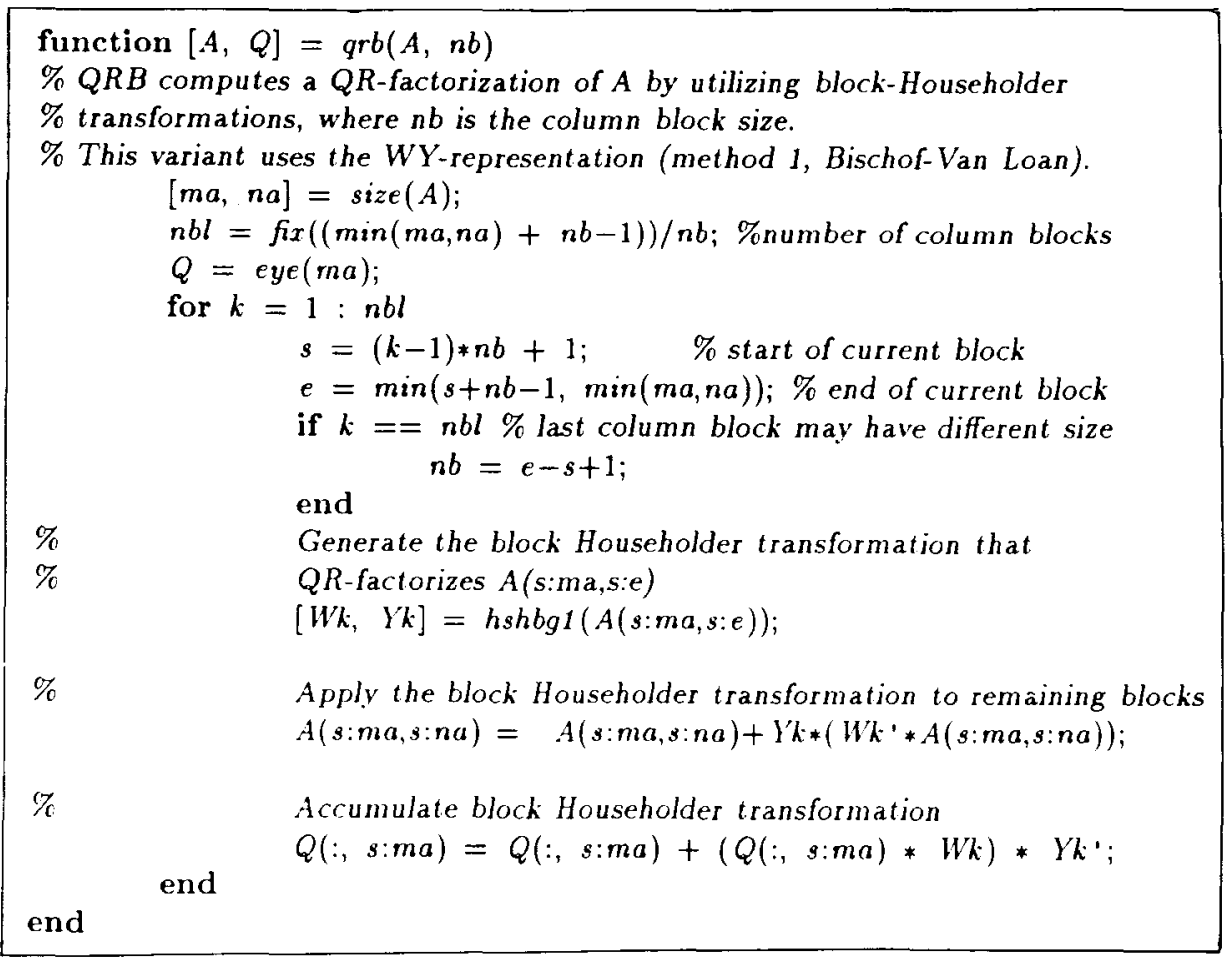

FIGLRE 11 MATLAB function for block ok factorization.

(a) NLAB implementanion is mumbered comsecomlively. Becalist: the algorithm only uses nearest-

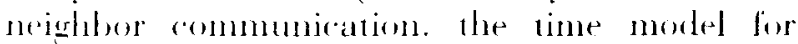
muli-hop communication is never involved and the mode numbering does not allece lle results. similar diagrame for the block torus matrix mulaiplication are shown in Firures 21 and 22. In whis example we have a more complex communication

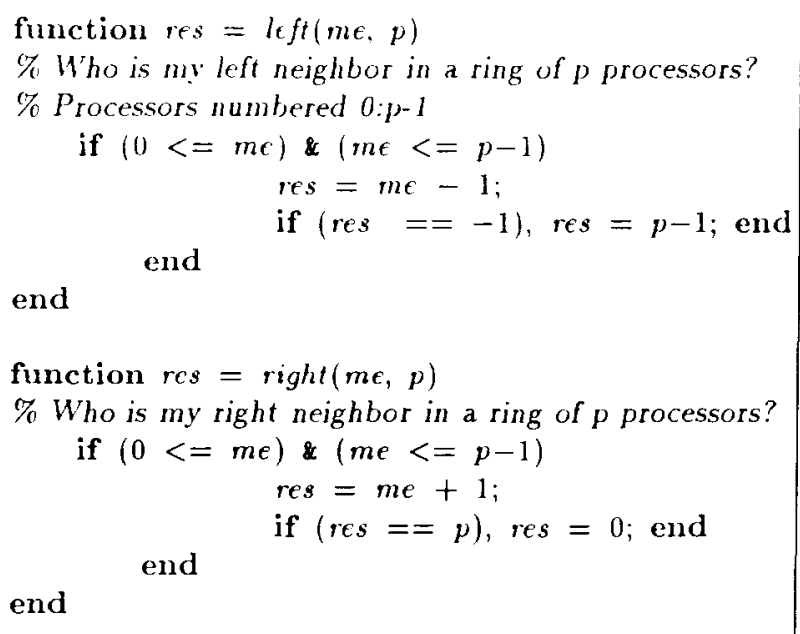

FIGLRE 12 Topology functions lor a ring of processols. pattern. but sill the communication paths ol the CONLAB simulation are accurate. The overhead from the tracing facility on the iPSC:2 is more visible in Figure 21 than in Figure 19. Both these examples do non une a communication chamel of the ipsci/2 for more than one mensage. This means that we will not encounter any communio'ation ehannel contention. At present. contention in the commonication system is not modeled in CONLAB. Therelore. ihe resulom from a simulation in CONLAB can be too optimistic for alanrithms that involve contention in the seal communication syotem. Honever. our experiences shon that we still gret a lave picume of the relative parallel performande when elangeing the size of the problem or the size of the scalable DNIM architecture.

\section{CONCLUSIONS AND FUTURE DEVELOPMENT}

Alerorithm development for D.MM architertures by using the CONLAB emiromment has been illustrated he a wo examples concerning matrix computalions. The algorithm derign procestes follons a development methorologr for DNMI algorishms that is based on different levels of abstraction of 


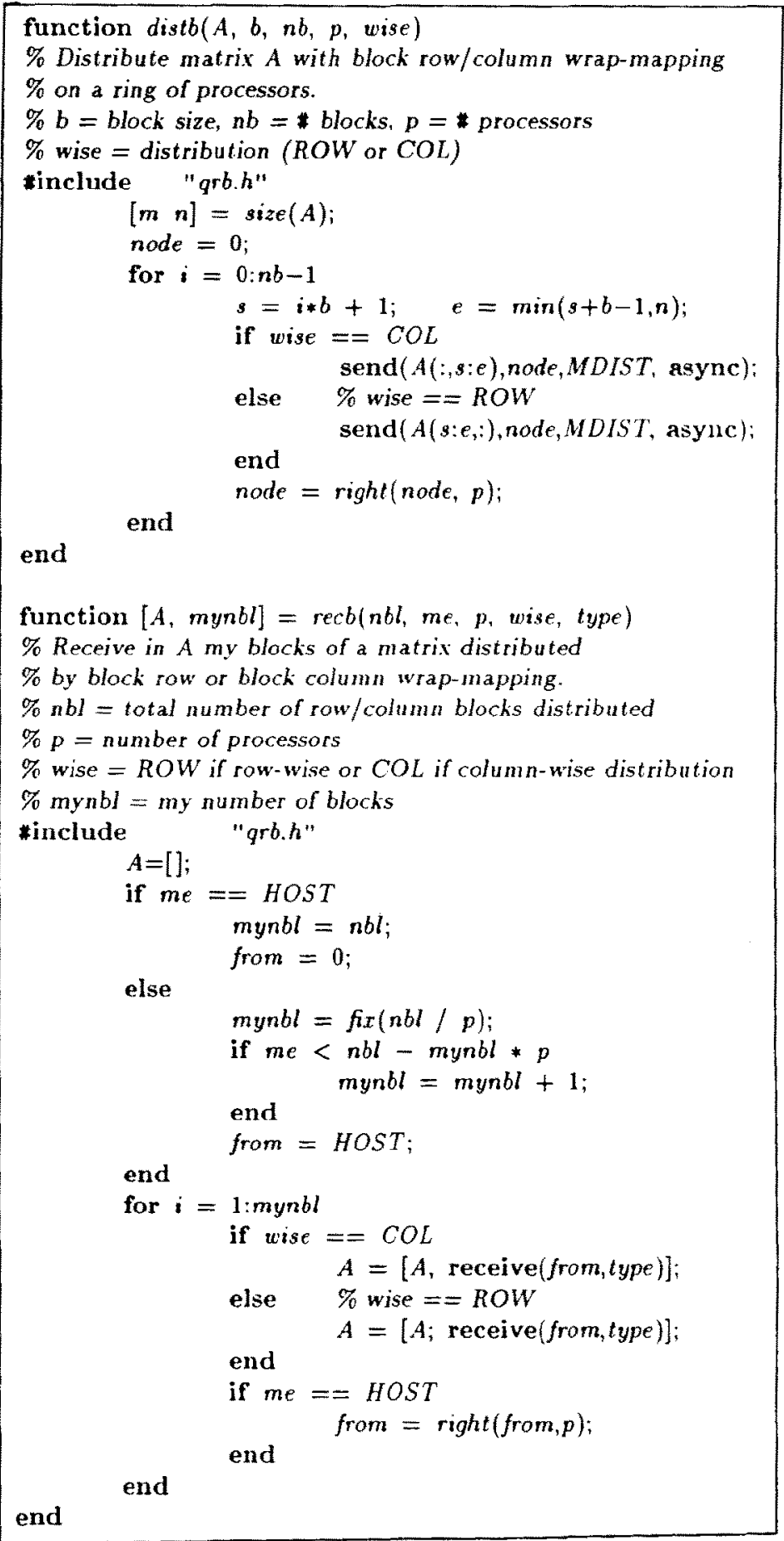

FIGURE 13 Communication functions for distributing and receiving block wapmapped data.

the problem, the target architecture. and the CONLAB language itself. Different tools and wavs for debugging algorithms and interpreting and evaluating the results have also been discussed.

CONLAB has extensively been used in parallel computing courses at our Lniversity. Further the parallel algorithms presented $[1.2,15]$ have first been designed in CONLAB and then explicitly translated by hand to Fortran or C. By designing and experimentally verifying the parallel algo- 


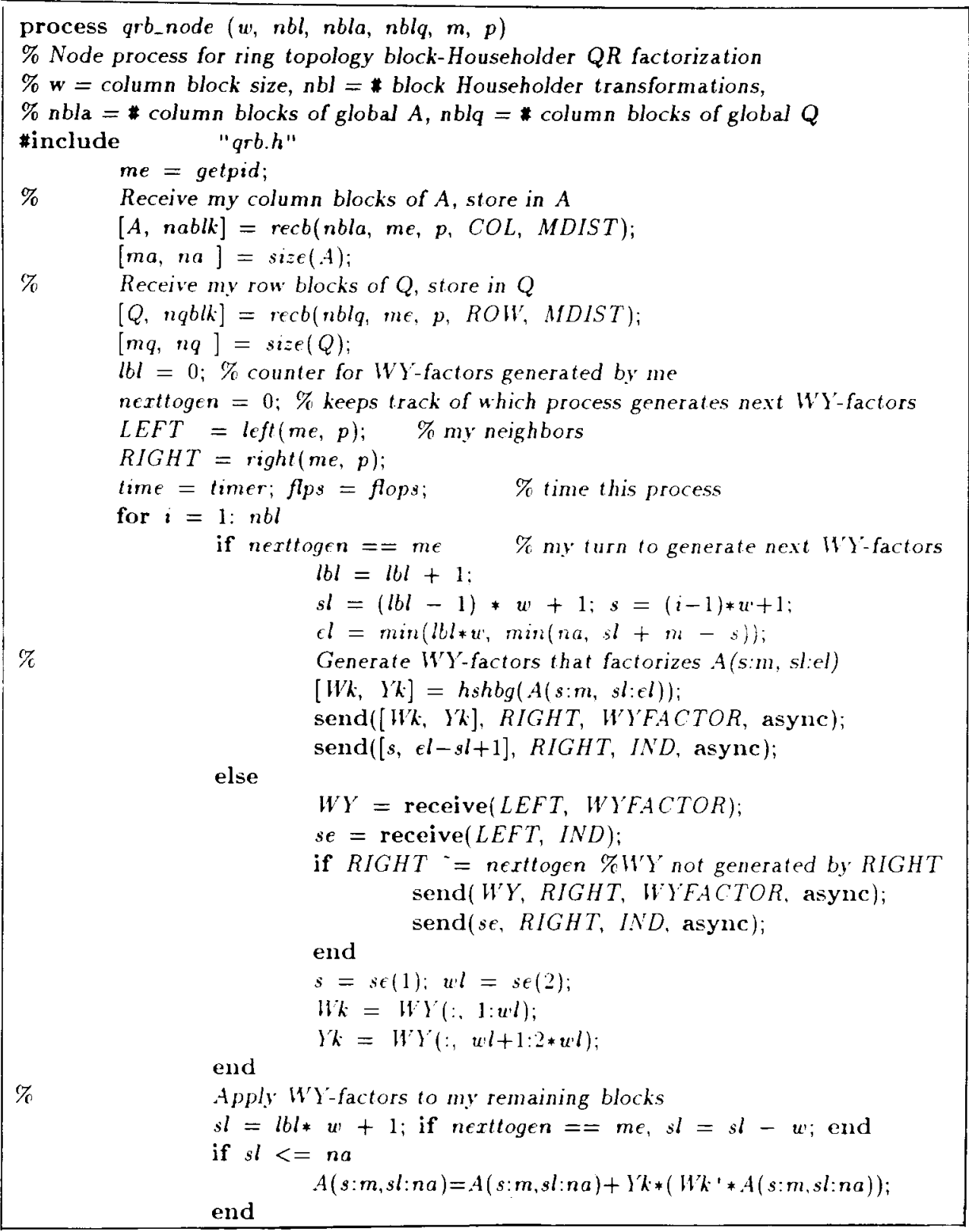

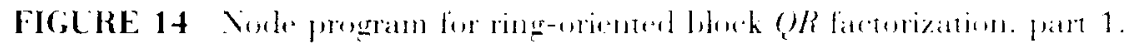

Table 1. Real and Simulated Performance Results of Torus Matrix Multiplication

\begin{tabular}{|c|c|c|c|c|c|c|c|}
\hline \multicolumn{2}{|c|}{ Torms $C=A \cdot B$} & \multicolumn{3}{|c|}{$i P S C / 2$} & \multicolumn{3}{|c|}{$(O N L A B$} \\
\hline$n$ & $P$ & $T_{p}$ & $S_{p}$ & $E_{p}$ & $T_{P}$ & $\dot{s p}$ & $E_{p}$ \\
\hline \multirow[t]{4}{*}{128} & 1 & $10.67+$ & 1.0 & 1.00 & $8 .+2$ & 1.0 & 1.00 \\
\hline & + & 2.29 & 4.7 & 1.16 & 2.21 & 3.8 & 0.95 \\
\hline & 16 & 0.63 & 17.1 & 1.07 & 0.6 .5 & 12.9 & 0.80 \\
\hline & $6+$ & 0.19 & 5.5 .6 & $0.8 ?$ & 0.30 & 28.2 & $0 .+4$ \\
\hline \multirow[t]{4}{*}{256} & 1 & 88.07 & 1.0 & 1.00 & $0 ?: 2+$ & 1.0 & 1.00 \\
\hline & $t$ & 21.58 & +.1 & 1.02 & 17.22 & 3.9 & 0.98 \\
\hline & 16 & +.66 & 18.9 & 1.18 & $+6 ?$ & $1+. t$ & 0.90 \\
\hline & $6+$ & 1.27 & 69.5 & 1.09 & $1.5+$ & +3.7 & 0.68 \\
\hline \multirow[t]{4}{*}{384} & 1 & - & - & - & 226.79 & 1.0 & 1.00 \\
\hline & + & $7.2 .50+$ & 1.0 & 1.00 & 57.02 & 3.9 & 0.98 \\
\hline & 16 & 15.99 & $4 . \overline{5}$ & 1.13 & 15.20 & $1+.9$ & 0.93 \\
\hline & $6+$ & +.08 & 17.8 & 1.11 & $+.5 \overline{5}$ & +9.8 & 0.78 \\
\hline
\end{tabular}


\% Accumulate block Householder transformations

if $m q^{-}=0$

end

$$
Q(:, s: n q)=Q(:, s: n q)+(Q(:, s: n q) * W k) * Y k^{\prime}
$$

end

nexttogen $=$ right $($ nexttogen,p $) ; \%$ next process to generate $W Y$ factor

total $=$ timer - time;

arith $=($ flops - fips $) *$ floptime;

com = total - arith;

disp ('Time pid $='$, me, ' Total $=$ ', total,, Arith $=', \ldots$

arith, ' Com $=$ ', com)

$\%$ Results to the host

for $i=1$ : nablk

$\operatorname{send}(A(:(i-1) * w+1: \min (i * w, n a)), H O S T, A R E S$, async $)$;

end

for $i=1: n q b l k$ send $(Q((i-1) * w+1: \min (i * w, m q),:), H O S T, Q R E S$, async $)$;

end

end $\%$ process bqr-node

FIGURE 15 Vode program for ring-oriented block ok factorization. part 2.

\begin{tabular}{|c|c|}
\hline \multicolumn{2}{|c|}{$\begin{array}{l}\text { \% Host id is used together with ParaGraph } \\
\text { \% to distinguish it from the nodes }\end{array}$} \\
\hline *define $H O S T$ & -32768 \\
\hline \multicolumn{2}{|l|}{ \% Message types } \\
\hline *define MDIST & 20 \\
\hline \#define WYFACTOR & 30 \\
\hline \#define $I N D$ & 40 \\
\hline *define $A R E S$ & 50 \\
\hline \#define $Q R E S$ & 60 \\
\hline \multicolumn{2}{|l|}{ \% Type of matrix } \\
\hline \#define $R O W$ & 100 \\
\hline \#define $C O L$ & 110 \\
\hline
\end{tabular}

FIGCRE 16 Header file. ring.h. for ring-oriented block $Q h$ fictarization.

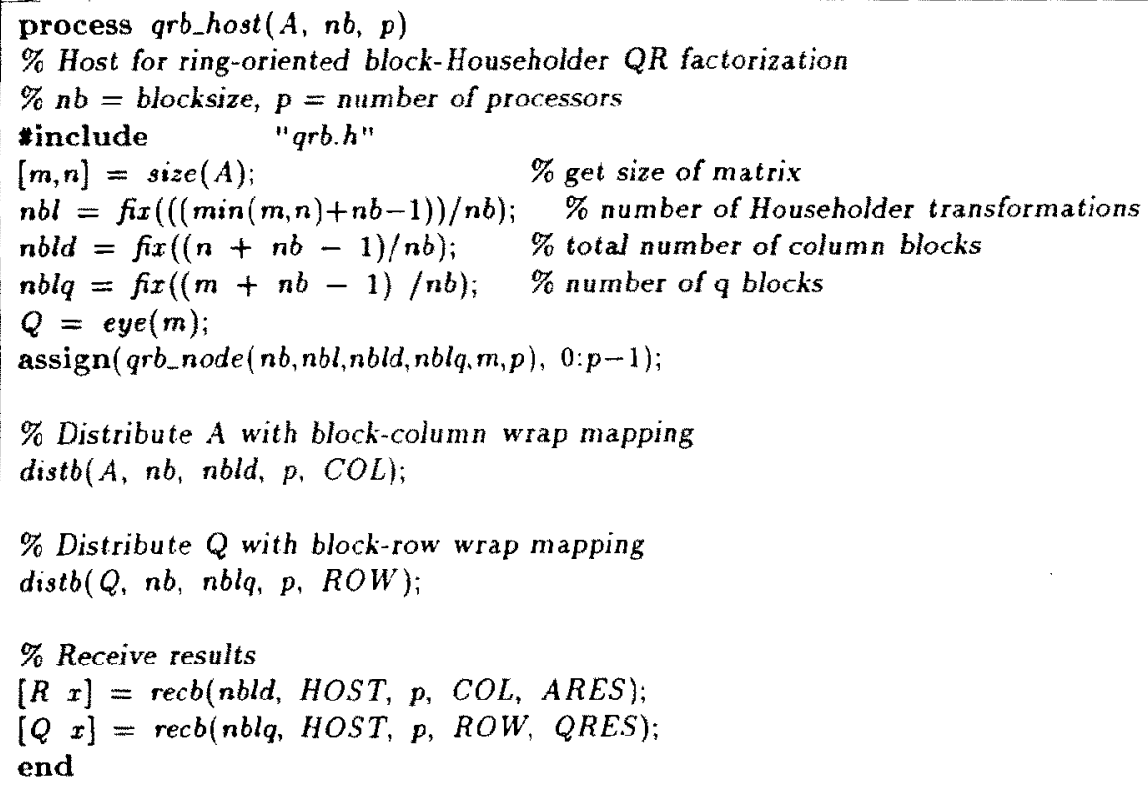

FIGLRE 17 Host program for ring-oniented block $Q R$ factorization. 
Table 2. Real and Simulated Performance Results of Ring-Oriented Block $O R$ Factorization

\begin{tabular}{|c|c|c|c|c|c|c|c|}
\hline \multicolumn{2}{|c|}{$\operatorname{Ring}() R$} & \multicolumn{3}{|c|}{ iPSC/2 } & \multicolumn{3}{|c|}{$(0 . \backslash L .1 B$} \\
\hline$m=n$ & $p$ & $T_{p}$ & $\therefore$ & $E_{p}$ & $T_{p}$ & $s_{p}$ & $E_{p}$ \\
\hline \multirow[t]{5}{*}{$6+$} & 1 & $5 .+06$ & 1.0 & 1.00 & $5 .+62$ & 1.0 & 1.00 \\
\hline & + & 1.58 & $3 .+$ & 0.86 & 1.71 & 3.5 & $0.8 ?$ \\
\hline & 8 & 0.9 .5 & 5.7 & 0.21 & 0.93 & 5.9 & $0.7+$ \\
\hline & 10 & 0.64 & $8 . \overline{5}$ & 0.5 .3 & 0.60 & 9.1 & 0.57 \\
\hline & 32 & $0 .+9$ & 11.0 & $0.3+$ & $0 .+2$ & 12.5 & 0.39 \\
\hline \multirow[t]{6}{*}{128} & 1 & $+3: 2: 3$ & 1.0 & 1.00 & +2.23 & 1.0 & 1.00 \\
\hline & + & 11.26 & 3.8 & $0.9+$ & 11.28 & 3.8 & $0.9+$ \\
\hline & 8 & 6.22 & 6.8 & 0.3 .5 & 6.14 & 6.9 & 0.80 \\
\hline & 16 & 3.69 & $11 .+$ & 0.71 & 3.50 & 11.9 & $0.7+$ \\
\hline & 32 & $2++$ & 17.3 & $0 . \overline{3}+$ & $2 \cdot 26$ & 18.7 & (0.58 \\
\hline & $6+$ & 1.82 & $2: 3.3$ & 01.30 & 1.50 & 20.0 & $0 .+2$ \\
\hline \multirow[t]{6}{*}{$2+11$} & 1 & $2^{-}+70$ & 1.0 & 1.00 & $2 ?++11$ & 1.0 & 1.00 \\
\hline & + & 70.92 & 3.9 & 11.97 & 70.89 & 3.9 & $0.9 ?$ \\
\hline & 8 & 37.20 & $7+4$ & 11.92 & 37.11 & $7 .+$ & 0.9 .2 \\
\hline & 16 & 20.73 & 1.3 .3 & 0.83 & 20.28 & $13 . \overline{3}$ & $0.8 \overline{5}$ \\
\hline & 32 & $12+3$ & 22.1 & $\left(0.0^{0}\right)$ & 11.87 & 23.1 & 0.7 .2 \\
\hline & 64 & 8.28 & 33.2 & 0.52 & 7.61 & .36 .1 & 0.50 \\
\hline
\end{tabular}

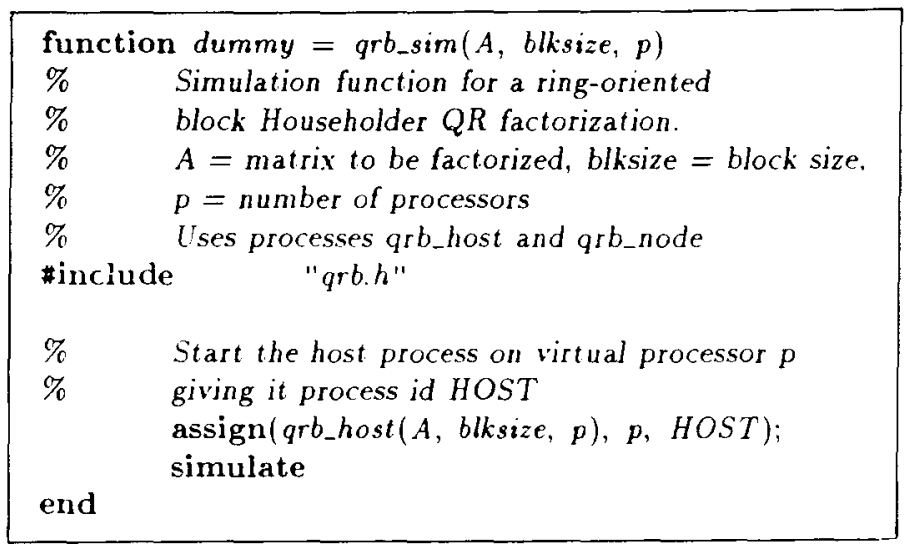

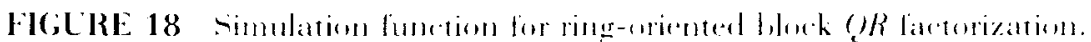

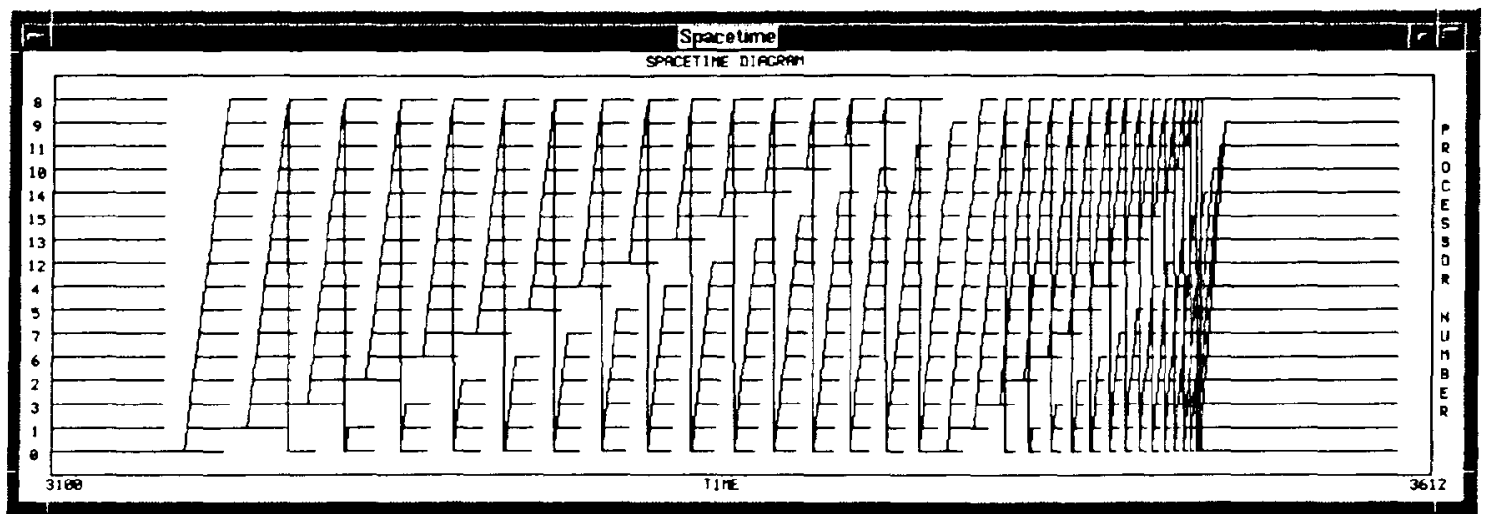

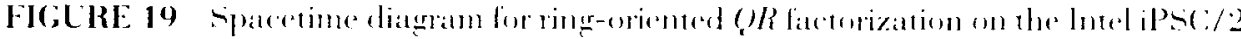
hyperculne. 


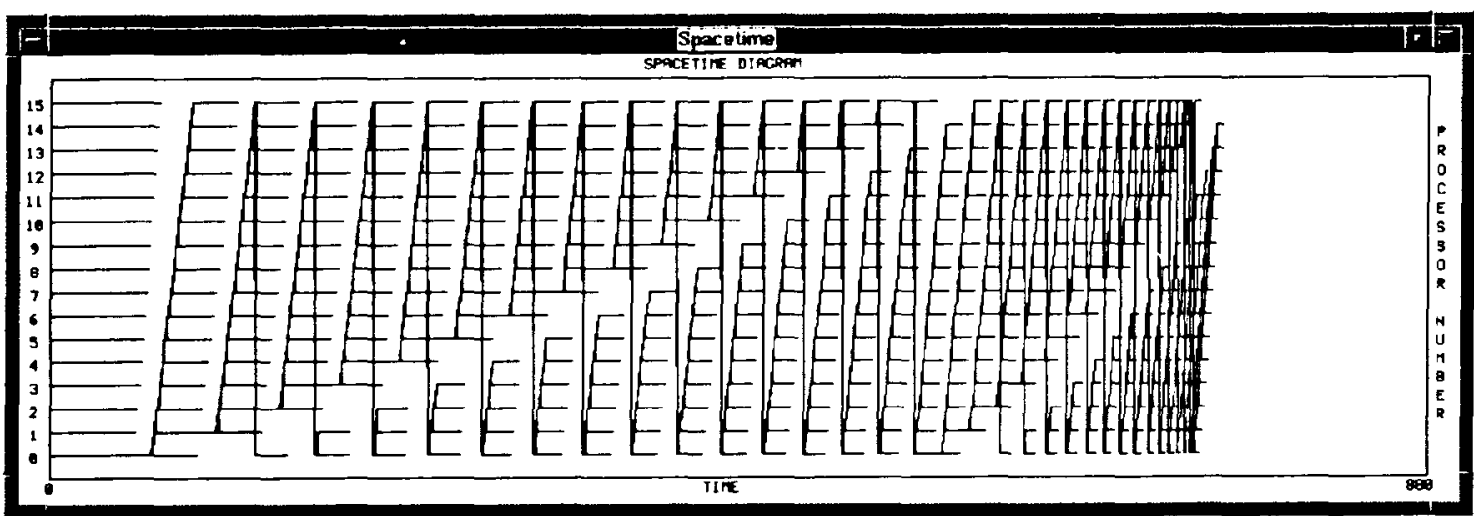

FIGURE 20 Spacetime diagran for ring-oriented $O R$ factorization simulating the iPSC/2 in CONLAB.

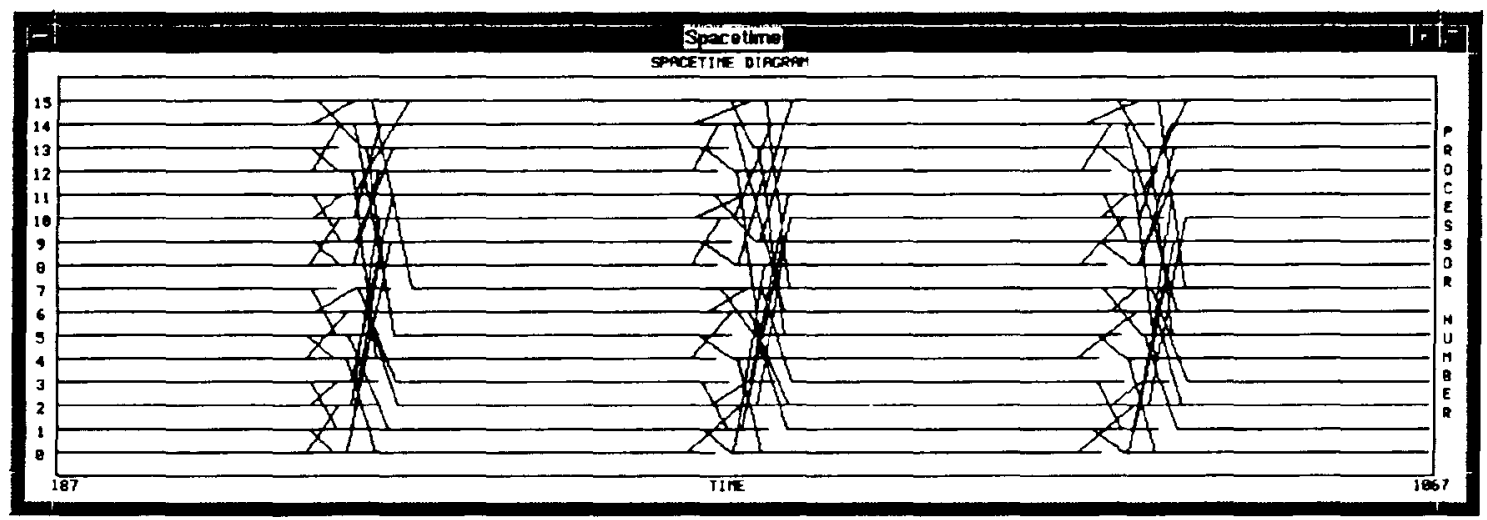

FIGURE 21 Spacetime diagram for block torus matrix multiplication on the Intel iPSC/2 hypercube.

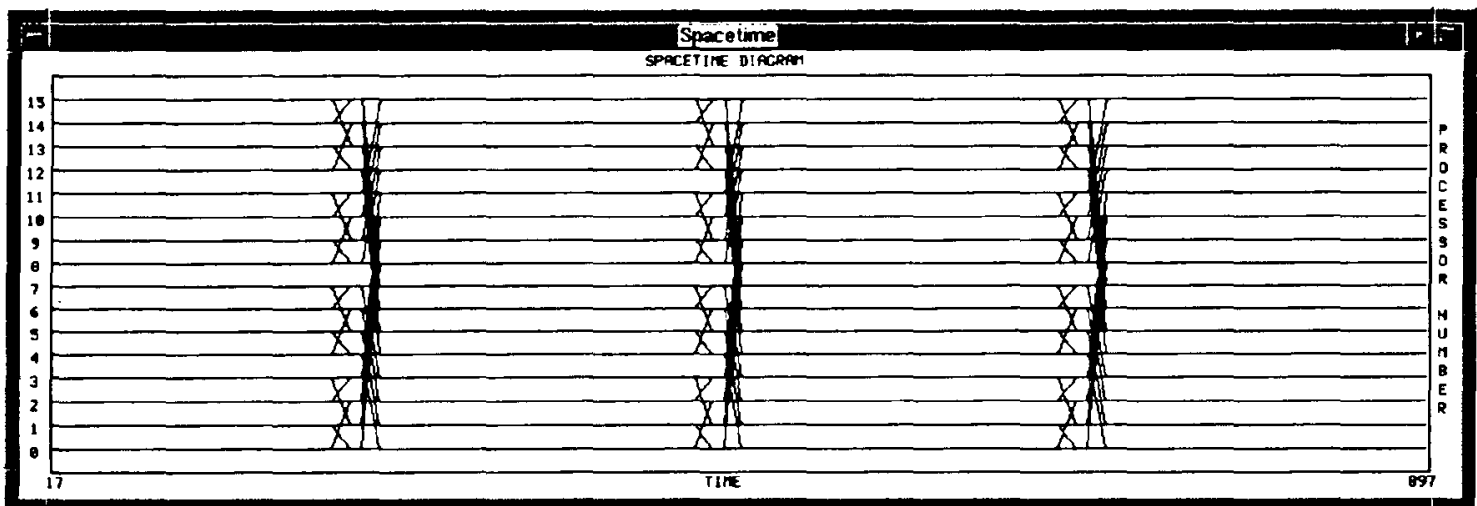

FIGURE 22 Spacetime diagram for the block torus matrix multiplication simulating the $\mathrm{iPSC} / 2$ in CONLAB. 
rithms in CONL AB the eiticiency and the quality of the development process have been improwed inpreciably.

The linture dirtations of develepentent of (:O) $L A B$ are awofold. Fint. The introluction of time

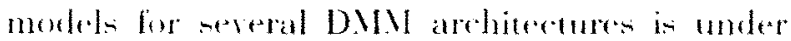

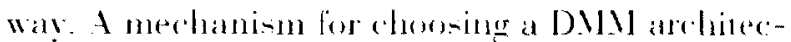
ture and corresponding architesture paramelex's exists atrearly. Second. and in parallel. the develrpment of a (O) NLAB compiler translator hat tarted. It akes a D.MII algorithm in CONL.MB

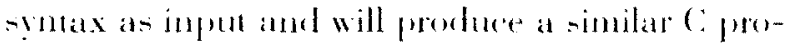

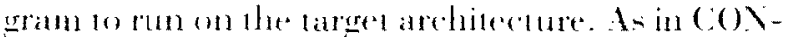
LAB. he mumerical compmations will be hated on L.APAC 3 . Further the commonication will be based on efliedent and portable impleme'nations of enploge and communication lunetions greesections 3 andel $t$

\section{ACKNOWLEDGMENTS}

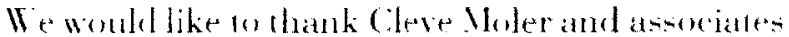
at The llathworks Ine for creating MATLAB. nithout whose eximence (COXLAB would never even have been thought of. Financial -uppert has: frecen received from the swedinh Board of Terhnial Development NCTEK under contraet STL 89-0.25.78P.

\section{REFERENCES}

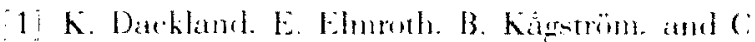
Van Lean. "Parallel hack matrix factomizations

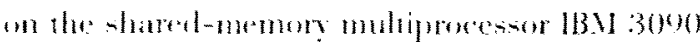

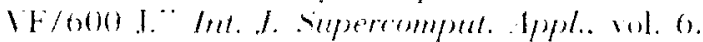
1). $69-9-9.1902$

2. K. Darkland and E. Elmmoh. "Pamallellhok ma-

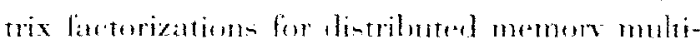

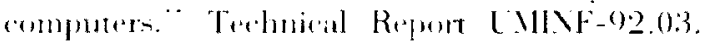

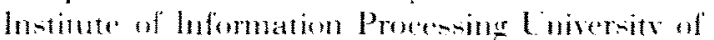
linca. s-901 8: Limeâ. Siweden. 1902.

3 E. Anderom, Z. Bai. C. Bischof, I. Demmel. I.

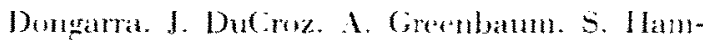
maling. A. Mekenmes. S. O-trouchor. and D.

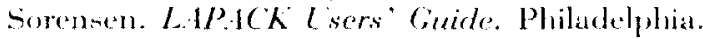

P. L: Sinciety for Indentrial and Ipplind Mathemanirs. Sll.11 Pullixations, 1002.

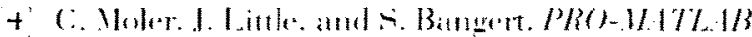
Cser's Givele. The Mathworki lore. 1987.

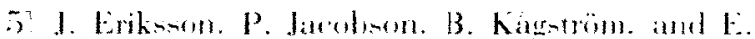

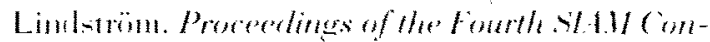

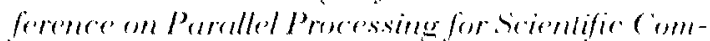

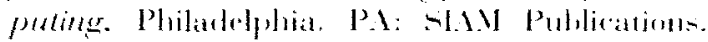
1090. $91 \%+00-+12$.

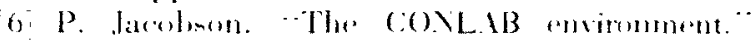

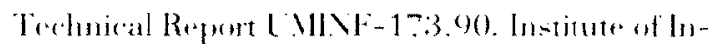

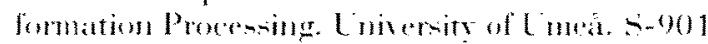
87. l meá. sweden. 190().

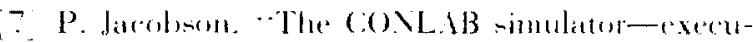
tion and as herbling of patrallel rompmations."

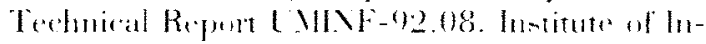

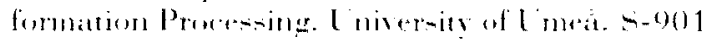
8: L Lmea sueden. May 1602.

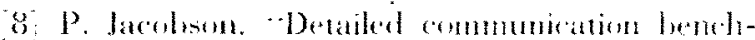
matke of Intel ipsci/2 and iPse:/800 hyper-

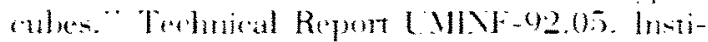

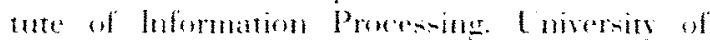

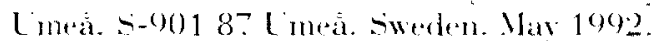

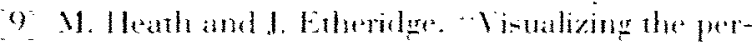
fomanow of paratlel programs." ILEE sofnare. wh. 8. pr. 20-39. 1001

10 G. A. G*ist. M. T. Meath. B. W. Prten and P. II.

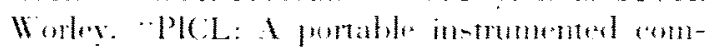
munication library." Technisal Report OR.SL/ TW-11130). Oak Ridere Vatimal Labonatory. 1000

11 L. Surder. Lamgurgere amel Compilers for Peurellel Completing. Boston. M1. MIT Press. 1990. ple. $+70-+89$

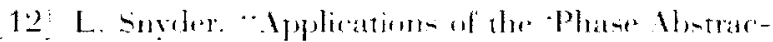
tions for portable and scabable prongramminge, Teluteal Repert. Deparment af Computer bej-

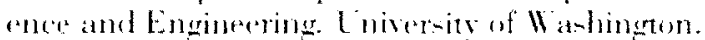
FR-35. Seattle. Washington 98195. LS1. 1900.

13) V. Cherkariky and R. Smih. "Efficient matyme and implementation of matrix algerithen on a bopereulue." I. Sepercomplat. vol. 2. pp. 7-27. 1988.

14 G. Golub amd C: Ian Loan. Metrix Computalionss. secend edition. Baltimore. MD: Jolur llopkins Cniversity Press, 1989. pp. 213-21t.

15) B. Kigstrom and P. Poromaa. Pistributed and shared memory block algorithms for the uriangular svlrester equation with sep ${ }^{-1}$ estimators."

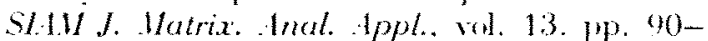
101. 1092. 

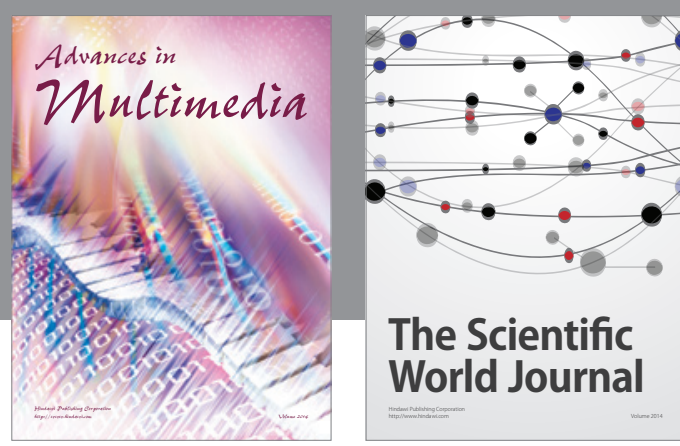

The Scientific World Journal
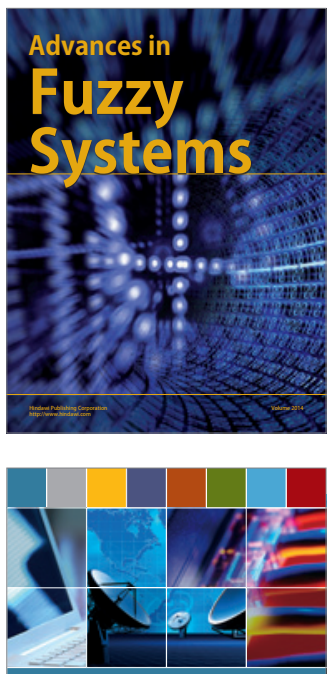

Computer Networks and Communications
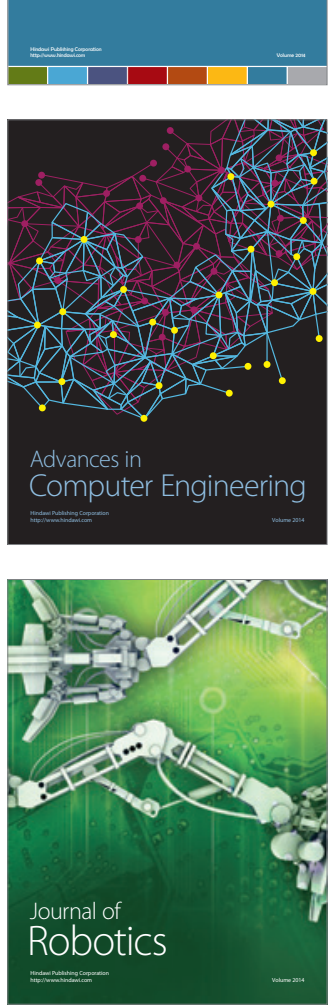
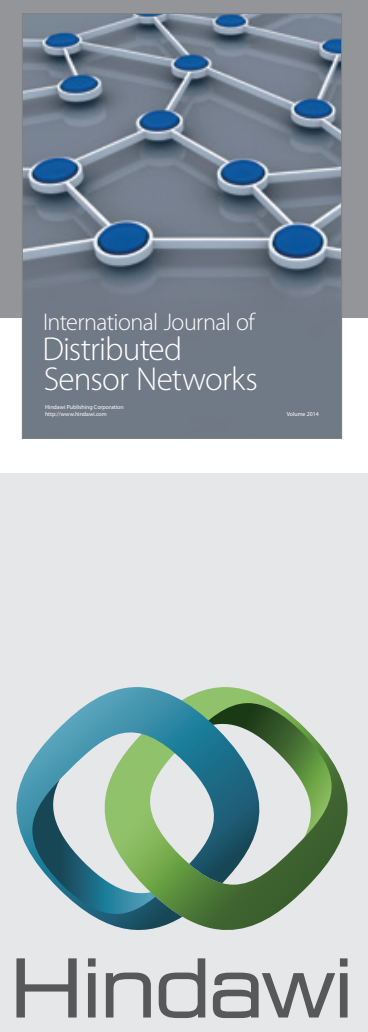

Submit your manuscripts at

http://www.hindawi.com
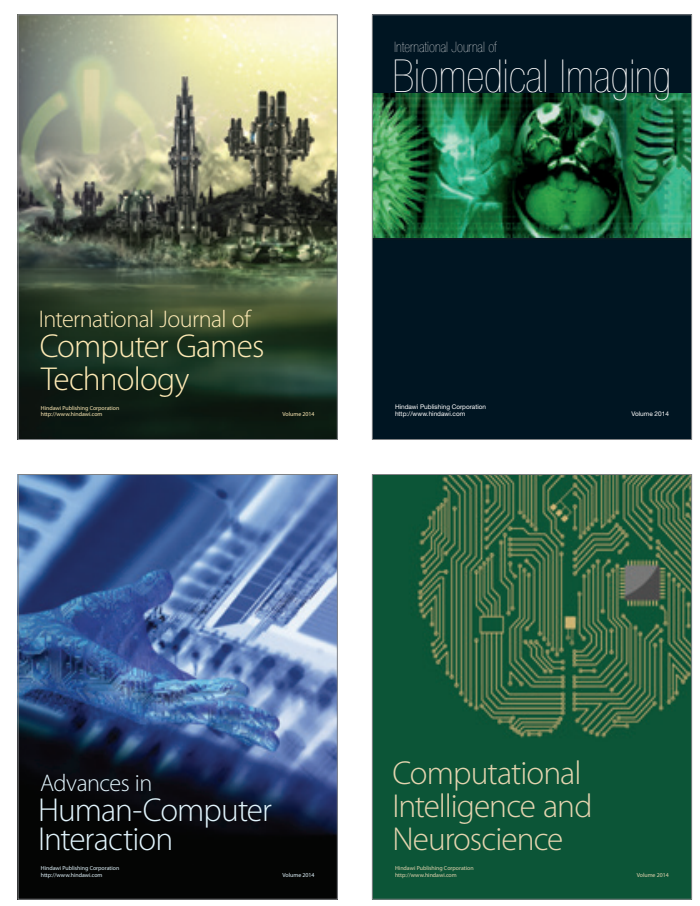
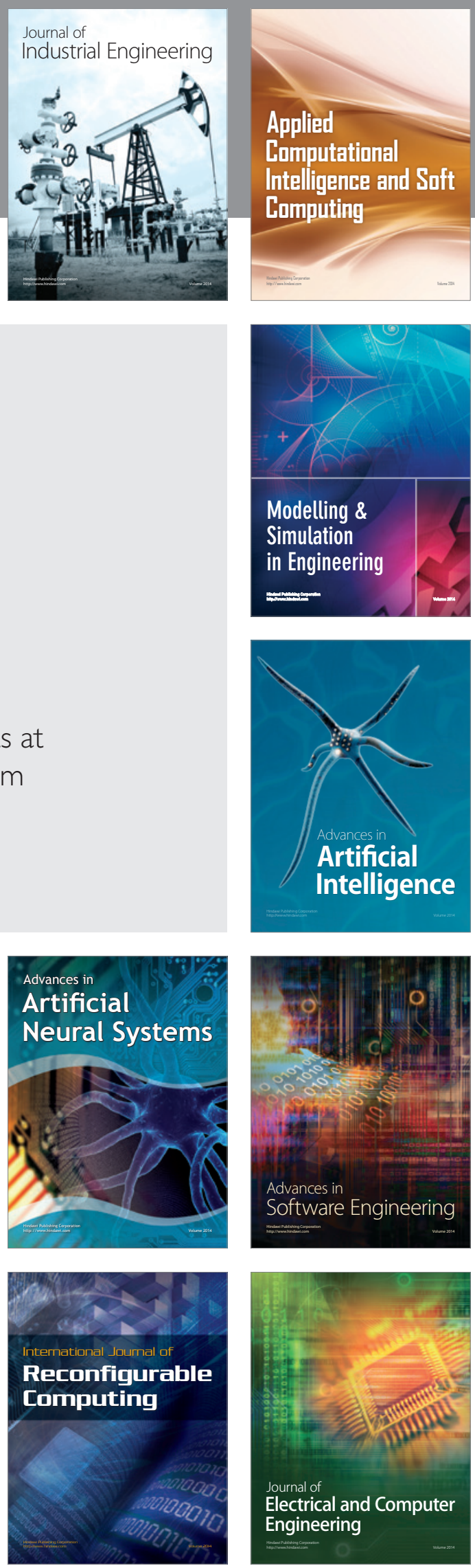